in dem sozialen Gefüge zu erkennen wären, auf die die zivile Konfliktbearbeitung einwirke.

Zum Abschluss der Tagung stellte Ariel Macaspac Penetrante sein Papier „Guns, Goons, and Gold as Burdens of a Fragile State - Dynamics and Mechanisms of SALW and Internal Conflict in the Philippines"vor. Darin untersucht der Autor anhand einer Studie zu den Philippinen den Zusammenhang zwischen schwacher Staatlichkeit und der Proliferation von Kleinwaffen. Als entscheidende Faktoren identifiziert er die horizontale und vertikale Gewaltenteilung sowie eine dominante männliche Waffenkultur.

Kommentar und Diskussion konzentrierten sich zum einen auf begriffliche Unklarheiten, fehlende Definitionen und den Verzicht auf einen friedenstheoretischen Bezugsrahmen. Weiterhin wurde die Verwendung eines westlichen Staatsbegriffs bei der Analyse nicht-europäischer bzw. nicht-westlicher Regionen problematisiert. Aus normativer Perspektive wurde in diesem Zusammenhang auf die Gefahren hingewiesen, die daraus erwachsen, den Staat als Ordnungsform absolut zu setzen und sein Potential zur Friedensgefährdung nach innen zu vernachlässigen.

Trotz der Heterogenität der theoretischen Ansätze und empirischen Phänomene, mit denen sich die Beiträge des Workshops beschäftigen, gelang es ihm deutlich besser als dem vorhergehenden, die Diskussionen auf die übergeordneten
Theoriefragen der Friedens- und Konfliktforschung zu beziehen. Es kristallisierten sich neben der anhaltenden Debatte über die Vereinbarkeit systemtheoretischer und handlungstheoretischer Ansätze für die Friedenstheorie drei wichtige Theorieprobleme heraus:

- Erstens ist das Verhältnis von Staat und Gewalt nach wie vor umstritten. Es scheint die Gemeinschaft der Friedens- und KonfliktforscherInnen weiter bezüglich der Bedeutung des Missbrauchs- und Entgrenzungspotentials staatlicher Gewaltmonopole auf der einen und der Anerkennung der friedensschaffenden Wirkung staatlicher Herrschaft auf der anderen Seite zu teilen.

- Zweitens bleiben theoretische Defizite bei der Erfassung der Interventionsproblematik sowohl in normativer (Legitimität und Legalität militärischer, ziviler, entwicklungspolitischer Interventionsentscheidungen) als auch in empirischer Hinsicht (Analyse der komplexen Interaktion von Strukturen, Prozessen und Akteuren).

- Insbesondere die Analyse von Hassreden, Massenmedien und Legitimationsmustern am Beispiel des Terrorismus bzw. der Terrorismusbekämpfung verdeutlichte drittens die zentrale Rolle von Sprache und Kommunikation bei Eskalations- und Deeskalationsprozessen in Konflikten sowie die mangelhafte Integration entsprechender Ansätze in die Theoriebildung der Friedens- und Konfliktforschung.

\title{
Macht, Recht, Frieden ${ }^{1}$
}

\author{
Egon Bahr ${ }^{2}$
}

1984 konzentrierte sich das ISFH auf den Bericht der PalmeKommission, der unter dem Titel „Gemeinsame Sicherheit“ zu der damals noch ungewohnten und umstrittenen Erkenntnis gekommen war, dass im Zeitalter gegenseitig gesicherter Zweitschlagsfähigkeit durch strategische Atomwaffen Sicherheit nur mit dem potenziellen Gegner zu erreichen ist und nicht mehr gegen ihn. In der damals bipolaren Welt konnten sich nicht nur die Regierungen, sondern auch die Wissenschaftler ein Ende der Sowjetunion nicht vorstellen.

Die Deutsche Einheit, die Implosion der Sowjetunion, das Ende des Wahrschauer Paktes beendete die Blockkonfrontation. Das Risiko eines apokalyptischen Krieges sankt gegen Null, aber die Realität vieler kleiner Kriege rückte die Vision eines dauerhaft gesicherten Friedens in unbestimmte Ferne. Aus der Blockdisziplin entlassen, schienen begrenzte Gewaltanwendungen wieder kalkulierbar. Das ISFH stellte fest: „Vier Jahre seit der säkularen Zäsur von 1989 zählt Europa ein vielfaches an Kriegstoten als in den vier Jahrzehnten der Ost-West-Konfrontation.“ Das Ergebnis unserer Überlegungen - in Anknüpfung an das kollektive Grundkonzept der UN - veröffentlichten wir 1993, ein Modell für eine „Europäische Sicherheitsgemeinschaft als

1 Manuskript eines Vortrages an der Universität Hamburg am 8.7.2008 zum Gedenken an Dieter S. Lutz, ehemaliger Direktor des IFSH und Begründer dieser Zeitschrift.

2 Bundesminister a.D., ehemaliger Direktor des IFSH.
Garant für Sicherheit und Frieden“. Die Leitidee meinte die Ablösung des Rechts des Stärkeren durch die Stärke des Rechts. Diese Maxime ist seither international geläufig geworden. Das Urheberrecht hat meiner Erinnerung nach eine Mitarbeiterin des Instituts. Der Spannungsbogen von Macht über Recht zum Frieden ist wie man sieht, bis heute aktuell geblieben.

Die Welt des Jahre 2008 ist mit der vor 18 Jahren kaum mehr vergleichbar. Russland ist nach einer Periode chaotischer Schwäche zu nationalem Selbstbewusstsein zurückgekehrt, basierend auf seinem Energiereichtum bei wachsendem Weltmarktbedarf und steigenden Preisen. Es ist das einzige Land, das über die atomare Zweitschlagsfähigkeit verfügt, obwohl die Abschreckung theoretisch weiter gilt, die Washington und Moskau pflegen, ohne von ihr abhängig zu sein. China ist Weltmacht geworden mit einer Wirtschaftskraft, die in überschaubarer Zeit die der USA übertreffen kann. In der Entwicklung seiner modernen Technologie steht Indien kaum nach. BRIC ist eine geläufige Abkürzung für die Giganten Brasilien, Russland, Indien und China geworden, die ihre Interessen abzustimmen begonnen haben. Amerika, Europa und Japan gehören nicht dazu. Klimaerwärmung und Bewahrung der Umwelt haben sich zu dem großen übergeordneten Thema des neuen Jahrhunderts entwickelt, sie werden die Weltmächte mittelfristig zu einer Zusammenarbeit nötigen, von der wir einen Vorge- 
schmack bekommen haben, als China wegen einer vergleichbar begrenzten Katastrophe Japan um militärische Hilfe bat.

Amerika hat zuerst die neue Weltlage erkannt und analysiert. Schon drei Jahre nach der Implosion der Sowjetunion hat „Die Kommission für Nationale Amerikanische Interessen“, nach unseren Begriffen überparteilich zusammengesetzt, ihre Arbeit begonnen. Ihr Bericht geht verständlicherweise davon aus, dass Amerika die einzige Supermacht bleiben will. Dazu muss sie militärisch-technologische Spitze bleiben, ihre Überlegenheit zur Uneinholbarkeit ausbauen, das Aufkommen jeder anderen Macht in Europa und Asien verhindern oder abschrecken. Dazu muss sie allein fähig sein, auch wenn Bündnisse nützlich bleiben; außerdem sollte Demokratie gefördert; und - nicht so vital - Menschenrechte berücksichtigt werden. Alle Präsidenten, Demokraten oder Republikaner, haben sich an diese Kriterien gehalten. Der kühlen und klaren Machtpolitik kann man Heuchelei nicht vorwerfen. Die militärischen Fähigkeiten Amerikas sind seither mit jedem Monat weiter gewachsen gegenüber allen anderen Staaten.

Die Frage wurde nicht diskutiert, warum Amerika sich nicht damit begnügte, als Sieger des Kalten Krieges seine Überlegenheit zu wahren und zu sichern. Eine Herausforderung seiner Macht gab es nicht. Stattdessen begann es eine neue Aufrüstung, das Gegenteil von Nachrüstung. Die ehemaligen Gegner waren ermattet oder schwach und die Freunde wollten die Friedensdividende genießen. Alle sahen sich einer neuen Rüstungswelle gegenüber, die um die Welt schwappte, über Europa und Russland auch Asien erreichte.

Der Weg Amerikas kann auch an zwei Doktrinen festgemacht werden: Von Bush (Vater) zu Bush (Sohn). Bush, der Ältere, bezeichnete Amerika als die überlegene Macht, die befähigt sei, die Welt in eine neue Weltordnung zu führen. Das wäre auf die Stärkung der UN hinausgelaufen, deren Ordnung Amerika als Erster unter Gleichen garantiert hätte. Bush, der Jüngere, nahm eine grundlegende Neuorientierung vor, als er mit seiner Sicherheitsdoktrin das Recht zur Kriegführung, auch präventiv, auch ohne Mandat der UN proklamierte.

Die neue Doktrin war nur die logische Konsequenz des politischen Entschlusses zu einem neuen gigantischen Rüstungsprogramm zu Lande, zu Wasser, in der Luft und im Weltraum, neue Atomwaffen eingeschlossen. Wir haben das, als es dem Streitkräfteausschuss des Senats im Juni 2001 vorgelegt wurde, nicht ernst genommen, weil es zu teuer schien und Vertragsbrüche verlangte und im Kongress scheitern würde. Der 11. September löste drei Monate später diese Probleme. Der demütigende Schock der Verwundbarkeit durch entstaatlichte Gewalt bescherte der Administration fast ohne Diskussion die Zustimmung des Kongresses zum Programm und seiner Finanzierung. Die Supermacht nutzte die Gelegenheit zu dem qualitativen Sprung der Uneinholbarkeit und zur Ausweitung von Einfluss durch Stützpunkte bis nach Zentralasien. Die NATO erklärte zum ersten Mal in der Geschichte den Bündnisfall, was Washington freundlich ignorierte und durch das Konzept der Willigen für die Unterstützung der jeweils anstehenden Missionen ersetzte.

Nun muss man gewiss verstehen, wenn Amerika sich in seiner Verantwortung nicht davon abhängig machen wollte, seinen Interessen $\mathrm{zu}$ folgen, ob andere Staaten mit jedenfalls sehr viel geringerer Verantwortung das billigten oder etwa warten sollten, bis Europa aus seinen jahrzehntealten Beschlüssen, mit ei- ner Stimme zu sprechen, vielleicht wirklich noch Wirklichkeit macht.

Immerhin ist festzustellen, dass es nicht sehr lange brauchte, bis aus einer einmütigen Solidarität mit Amerika, die bis nach Afghanistan trug, die erstaunliche Verweigerung wichtiger Verbündeter wurde, sich am Irakkrieg zu beteiligen. Natürlich war der deutsche Bundeskanzler nicht so stark, um Paris und Moskau und den Papst zu verführen; er war auch nicht mächtig genug, die Massen in London, Rom, Madrid und Warschau gegen den Willen ihrer Regierungen auf die Straße zu bringen. Darin drückte sich auch der europäische Zweifel an Sinn und Legalität des Ganzen aus. Das Resultat zeigte: Macht ohne Recht führt zu Krieg und nicht zum Frieden. In Umrissen zeichnete sich ab, dass amerikanische und europäische Interessen auseinander driften.

Robert Kagan, ein brillanter amerikanischer Analytiker, hatte in seinem Buch „Macht und Ohnmacht“ 2003 definiert, dass „sich Amerikaner und Europäer immer weiter auseinander entwickeln" und diese Unterschiedlichkeit mit dem Bild von Mars und Venus verglichen, von Macht und Sendungsbewusstsein gegenüber Schwäche und Sendungsbewusstsein. Auf der einen Seite Besitz und Einsatz militärischer Macht, wie eh und je in der Geschichte, auf der anderen Seite Abwendung von der Macht und Hinwendung zu einer in sich geschlossenen Welt des verhandelten Friedens durch Gesetze und Regeln. Damit beschrieb Kagan die Ziele einer europäischen Sicherheitsgemeinschaft, allerdings mit dem Irrtum, dass ein Gebäude des organisierten Gewaltverzichts in Europa sehr wohl die Macht der Schwachen bedeutet und ihre Regeln nicht in ein konfliktfreies Paradies, sondern zu friedlichen Regelungen führen müssten und würden. Kagan erkannte durchaus, dass eine solche Sicherheitsgemeinschaft eine multipolare Welt schaffen könnte und eine imperiale Neuordnung zu verhindern suchen würde. Das Bild der ersten Venus war ihm nicht geläufig: Eva hat Macht durch Überzeugung und Verführung des physisch stärkeren Adam ausgeübt.

In der Wirklichkeit erwies sich Europa als unfähig, seine Interessen zu bündeln, auch nur zu diskutieren. Es verhielt sich, als ob Brzezinskis Feststellung, Europa sei sicherheitspolitisch ein Protektorat der USA, auch in Zukunft gelten werde. Die Welt folgte der riesigen Rüstungswelle, die Washington ausgelöst hatte, Europa ließ sich hinreißen, den amerikanischen Vorgaben zu folgen, Qualität und Richtung der Modernisierung seiner Streitkräfte auf den globalen Einsatz neben den Amerikanern auszurichten. Wer da führt, ist klar und undiskutabel. Mit jedem Monat vergrößert Amerika die transatlantische Kluft, soweit seine technischen Fortschritte das erlauben. Es ist perpetuierte Demonstration der europäischen Ungleichheit gegenüber einer High-Tech-Armee, ausgerüstet für globale Kriegführung. Europäische Interessen würden Streitkräfte erfordern, die Stabilität für die Erhaltung des Friedens in Europa garantieren können.

Die große Tradition des Gewaltverzichts, diese erwiesene Stärke des Schwachen, ist verloren gegangen, und das große Thema der Abrüstung ebenfalls. Die Friedensgutachten der letzten zehn Jahre dokumentieren es. Die Urheberschaft für diesen Zustand unserer Welt liegt in den USA, eine Mitschuld durch Schwäche bei den Europäern.

Parallel dazu hat Amerika unwillentlich eine andere Entwicklung gefördert. Zwischen 2002 und 2006 reicht die kurze 
Zeitspanne, in der Washington auf eine unipolare Welt hoffen konnte. Keine Weltherrschaft, aber die Festigung seiner Vorherrschaft in der Welt. China, Indien, Japan und Russland sind, veranlasst oder gedrängt durch Amerika, auch zu militärischen Faktoren geworden, die Multipolarität de facto etabliert haben. Die erhaltene und noch immer nicht herausgeforderte militärische Überlegenheit Amerikas reicht nicht mehr aus, seine nationalen Interessen in Asien und im Mittleren Osten durchzusetzen, ohne die Interessen mindestens der neuen drei Großmächte zu berücksichtigen. In Asien ist Amerika nur noch eine unter anderen Mächten, um Stabilität garantieren zu können. Globalisierung ist nicht mehr gleichbedeutend mit Amerikanisierung.

Das ändert nichts an mindestens zwei Wirklichkeiten: Im Übergang von der lahmen Administration eines Präsidenten, der stolz darauf war, sich Kriegspräsident zu nennen, zu seinem Nachfolger, läuft die Rüstung auf vollen Touren und vergrößert den Vorsprung gegenüber allen Anderen. Zum Zweiten: Das Bewusstsein seiner Werte, seiner Macht und seiner Mission wird auch in Zukunft durch keinen Präsidentenwechsel verändert oder vermindert werden. Beide Wirklichkeiten darf man nicht übel nehmen. Die eine handelt von Hard-Power, die andere geht in die Soft-Power über und bildet den Hintergrund, auf dem Methoden, auch skrupellose, zur Durchsetzung politischer Ziele benutzt werden, heute wie gestern und bestimmt auch morgen.

In den Staaten ist gerade ein aufregender Film produziert worden. Er dokumentiert die Verführbarkeit der Öffentlichkeit durch Fälschungen und Lügen der Regierungen vom Vietnamkrieg über den ersten und zweiten Irakkrieg bis zur Gegenwart. Die elektronischen Medien mit ihren bewegten Bildern erzeugen eine Überzeugungskraft, die eine Macht darstellt, gegen die kritische Argumente nicht mehr wirken. Zumal die gedruckten Medien sich gezwungen sehen, das Fernsehen zu berücksichtigen. Journalisten haben schon immer abgeschrieben. In Situationen, die auf Spannung und Erhöhung der Spannung tendieren, wirken sie als Verstärker. Der Mainstream wird zu einem reißenden Meinungsstrom, gegen den einzelne Stimmen der Vernunft nicht mehr ankommen oder verhöhnt werden oder aus Angst vor wirtschaftlicher Existenzgefährdung nicht mehr formuliert werden. Bedrohungen werden aufgebaut und mit Bildern bewiesen, die nichts beweisen, bis sich durchsetzt, dass man sich schützen, die Gefahren für die eigenen Werte abwehren und notfalls verteidigen muss. Vor dem Krieg gegen Saddam Hussein wurde sogar die Glaubwürdigkeit des eigenen Außenministers zerstört. Wer den Frieden will, muss sich für den Krieg vorbereiten. Das alte Lied wird in vollendeter Manier modernisiert. Die Hoffnungen, Gewalt vermeiden zu können, suggerieren, dass diplomatische Bemühungen doch noch Erfolg haben könnten, bis der Ruf nach Krieg überwältigend wirkt. Der Wunsch nach Sicherheit und Frieden bereitet den Krieg vor. Die Mechanismen sind bekannt und wiederholen sich, während in Wahrheit der Krieg längst beschlossen ist.

Ich habe das einmal hautnah erlebt. Im Sommer 2002 hatte mich unser Botschafter Ischinger in Washington zu einem Mittagessen in seine Residenz eingeladen. Ich wollte gute Bekannte treffen, aus State Department, Pentagon, Sicherheitsrat und CIA. Wir sprachen so offen, wie in alten Zeiten unter Freunden. Es ging um Saddam Hussein. Ich lernte: Wenn der Präsident die Weisung gibt, sind wir fähig in zwei Wochen einen Schlag zu führen, um den Diktator zu entführen oder zu töten. Da dies auch mit dem Blick auf die Midterm-Elections, also die Zwischenwahlen im November mit einem zu großen Risiko verbunden ist, ist entschieden worden, einen richtigen Krieg vorzubereiten. Für den erforderlichen Aufmarsch von 500.000 Mann ist ein Zeitbedarf von sechs Monaten erforderlich. Das reicht aus, um durch diplomatische Aktivitäten in den UN vom Aufmarsch abzulenken. Nach Rückkehr habe ich den Bundeskanzler informiert, dass Amerika im Februar (2003) den Krieg gegen den Irak beginnen wird, mit oder ohne Mandat der UN. Ich hatte den Ramadan nicht berücksichtigt. Die Invasion begann erst im März.

In diesem Sommer habe ich gewissermaßen eine Art halbamtliche Bestätigung meiner Information mit sechsjähriger Verspätung erfahren. Der langjährige Sprecher des Weißen Hauses, McClellan hat in einem Aufsehen erregenden Buch die Geschichte seiner Täuschungen beschrieben; darin ist auch die Passage enthalten, dass bereits im Sommer 2002 begonnen wurde, „den Krieg durch Manipulation von Informationen aggressiv zu verkaufen“.

Die Realität zeigt also: Am Anfang steht der politische Entschluss. Der erwähnte Film zeigt nur die Methodik, eine kriegsunwillige Bevölkerung vorzubereiten und zu gewinnen. In unserem Medienzeitalter verlangt der Wille zur Täuschung des eignen Volkes die Täuschung der Welt. Weil der Glaube, dass die Kinder vom Storch gebracht werden, immer mehr schwindet und zwar nicht nur im christlichen Abendland, nimmt auch die Glaubwürdigkeit an die Motive ab, mit denen Amerika seine Machtinteressen begründet, verschleiert und Aktionen vorbereitet. Und dabei muss es nicht immer bis zur offen Gewaltanwendung gehen. Die Welt ist in der Regel schon erleichtert, wenn der Schmerz nachlässt und der Krieg vermieden wird.

Nun hat Robert Kagan ein neues Buch geschrieben. Es besticht zunächst durch die ungewöhnliche Offenheit, mit der er rückblickend die politische Rücksichtslosigkeit seines Landes beschreibt. Jeder europäische Autor müsste sich gegen den Vorwurf des Antiamerikanismus zu verteidigen haben, der die folgenden Zitate formulieren würde: „Die Vereinigten Staaten, obwohl traditionell auf die eigene Souveränität bedacht, waren stets bereit, sich in die inneren Angelegenheiten anderer Nationen einzumischen“..."Die Bereitschaft, seine Schlagkraft für verschiedenste Zwecke einzusetzen, reicht von der humanitären Intervention in Somalia und in Kosovo bis zur Erzwingung des Regimewechsels in Panama und im Irak. Zwischen 1989 und 2001 griffen die USA mehr und häufiger als jede andere Macht mit ihren Streitkräften im Ausland ein. Im Schnitt erfolgte alle 16 Monate eine bedeutsame militärische Aktion, wo es zur Beförderung amerikanischer Ideale und Interessen zweckmäßig schien“. Die Selbstverständlichkeit, mit der Ideale, Interessen und Zweckmäßigkeit gleichgestellt werden, ist beachtlich.

Ich zitiere weiter: „Seit dem 9. September 2001 haben die USA Stützpunkte in Zentralasien, in Afghanistan. Kirgisien, Pakistan, Tadschikistan und Usbekistan, dazu in Europa, Bulgarien, Georgien, Ukraine, Polen und Rumänien sowie auf den Philippinnen, in Dschibuti, Oman, Katar und natürlich im Irak eingerichtet. Die Erweiterung der NATO auf die drei baltischen Staaten sollte nicht vergessen werden.“

$\mathrm{Zu}$ den brutalen Eingeständnissen der bisherigen Praxis erinnert Kagan, dass „die demokratische Welt und die USA vorne weg, internationale großzügig mit Mitteln ausgestattete nicht- 
staatliche Organisation (NGOs) schulten und oppositionelle Parteien unterstützten, um durch Wahlrechtsreformen und in Mittel- und Osteuropa und in Zentralasien Regime zu Fall zu bringen. Prowestliche und demokratiefreundliche Politiker und Parteien haben mit organisatorischer und finanzieller Unterstützung geholfen, Autokratien in Georgien, in der Ukraine und im Libanon zu stürzen." Entsprechende Sorgen Putins scheinen Kagan nicht unangebracht, um NGO-Tätigkeiten in Russland zu beschränken und notfalls zu verbieten.

Er äußert auch Verständnis für das russische Gefühl der Demütigung, nach dem ihm die Hinnahme des wachsenden amerikanischen Einflusses „aufgezwungen“ wurde. Auf der anderen Seite findet er, dass „die NATO in dem Maße harmloser geworden ist, wie Russland aggressiver wurde“.

Kagans Blick in die Zukunft leitet sich von dem Krieg gegen Jugoslawien ab. Russland und China waren gegen die Intervention 1999 „nicht allein, weil die chinesische Botschaft in Belgrad von einem amerikanischen Kampfflugzeug bombardiert und Russlands ferne slawische Verwandte in Serbien zur Zielscheibe der NATO-Luftangriffe wurden. Als Russland im UN-Sicherheitsrat damit drohte, die Militäraktionen zu blockieren, umging die NATO die Vereinten Nationen kurzerhand, ermächtigte sich selbst zur militärischen Intervention und nahm Russland damit eine der wenigen internationalen Einflussmöglichkeiten. Auch weil hier gegen einen souveränen Staat interveniert wurde, der keine äußere Aggression begangen hatte, war der Krieg aus Moskauer Sicht ein klarer Verstoß gegen das Völkerrecht. "Diese Umwertung internationalen Rechts bezeichnet Kagan als Triumph und als Modell: Die internationale Gemeinschaft sollte das Recht proklamieren, im Falle gravierender Bürgerrechtsverletzungen gegen souveräne Staaten einzuschreiten und das als völkerrechtliches Prinzip verankern.

Gerade rechtzeitig bevor eine neue Administration beginnen kann, sich in Washington einzuarbeiten, entwickelt Kagan seine Auffassung, wonach „der Kampf auch nach dem Fall der Mauer weitergeht“. Nach dem Sieg der westlichen Freiheit über die kommunistische Unterdrückung kann und sollte die demokratische Welt nun ihre universellen Werte, Demokratie und Menschenrechte verbreiten, dazu auch Macht einsetzen und damit Andere zur Annahme dieser Werte und Prinzipien zwingen.

„Die Anhänger dieses liberalen Glaubensbekenntnisses können eine Außenpolitik und sogar Kriege, deren Zweck - wie im Kosovo - dem Schutz dieser Prinzipien gilt, auch dann gutheißen, wenn sie gegen verbrieftes Völkerrecht verstoßen. Schließlich hat sich selbst das postmoderne Europa schon im Namen dessen, was ihm als höhere Moral der Aufklärung galt, über die rechtlichen Feinheiten hinweggesetzt.“

Die Gegner sind also die Autokratien. Dazu gehören zunächst China und „Putins souveräne Demokratie“. Die neue Ära wird eine Zeit wachsender Spannungen und manchmal auch der Konfrontation zwischen den Kräften der Demokratie und denen der Autokratie sein. Dazu könnte es zu Erschütterungen entlang der europäisch-russischen Verwerfungslinie kommen, die eines Tages in eine Konfrontation münden. „Wenn die Ukraine eines Tages der NATO beitreten wollte, könnte das die Kampfeslust der Russen anstacheln“.

Das Gedankengebäude verbindet Logik mit Fantasie: Am Anfang steht ein Vision zu einem neuen Horizont. „Was einst unvorstellbar war, wird erst vorstellbar, dann erstrebenswert. Aus dem Wunsch, wird ein Ziel, aus dem Ziel ein Interesse“, soweit Kagan. Das Interesse muss dann auch verteidigt werden, also wenn erforderlich durch Angriff gegen Unbelehrbare.

Dieser Kampf um die künftige Weltordnung verlangt ein Instrument. Der UN-Sicherheitsrat erfüllt diesen Zweck nicht. Kagan schlägt dafür vor, einen „Bund der Demokratien“ ins Leben zu rufen. Er soll die Kräfte der westlichen demokratischen Nationen mit denen aus Asien verbinden und neben den Vereinten Nationen die erforderlichen Aktionen legitimieren. So könnte die amerikanische Hegemonie durch die Überwindung der Autokratien gesichert werden. Ich füge hinzu: Es wäre bestimmt ein angenehmer Nebeneffekt, wenn auf diese Weise die unaufhaltbare Multipolarität unter dem Banner „Demokratie und Menschenrechte" unterlaufen wird. Das ist eine bestechende Idee, der Welt letztlich den Frieden zu bringen durch die Legitimierung von Macht nach amerikanischem Recht.

So interessant diese Vision zu einem neuen Horizont ist, so alarmierend wird sie, sobald daraus operative Politik entwickelt wird. Ich hatte kürzlich Gelegenheit, einen Amerikaner zu hören, der in einer Schlüsselfunktion der NATO tätig ist. Der analysierte, dass nach der Stabilität in Europa die Transformierung der übrigen Welt ansteht. Das NATO-Instrument der erweiterten Partnerschaft könnte auf Australien, Neuseeland, Japan und andere Staaten angewendet werden. Die Frage liegt nahe: Befinden wir uns noch in dem Stadium, wo aus dem Wunsch ein Ziel oder schon im Übergang, wo aus dem Ziel ein Interesse wird? Immerhin hat Senator McCain als Teil seiner Außenpolitik als Präsident die Gründung einer „League of Democracies“ angekündigt für Länder, die noch keine Demokratie haben.

Die Veränderung der Welt anstelle der Sicherung der Stabilität entspricht der Tradition amerikanischen Denkens und Fühlens. Alle Präsidenten, die wir in den letzten 60 Jahren erleben konnten, sind von tiefer unangefochtener Überzeugung getragen worden: Was gut ist für Amerika, ist gut für die Welt. In unterschiedlicher und individueller Ausprägung teilten alle Hausherren im Weißen Haus das amerikanische Sendungsbewusstsein und alle folgen den strategischen Interessen ihres Landes. Auch wenn die Hoffnung berechtigt ist, dass dies künftig nicht nach der Machart des jetzigen Amtsträgers geschehen wird, wird sich die Grundrichtung nicht ändern: Festigung und Ausweitung des globalen Einflusses. Obama und McCain verbindet die Vorstellung von der geschichtlichen amerikanischen Bestimmung. Praktisch wird es selbstverständlich ein gewaltiger Unterschied sein, wie das amerikanische Grundgefühl übersetzt wird: In die Politik einer Weltordnung, in der die USA Erster unter Gleichen sind oder in eine Weltvorherrschaft mit der NATO als gewaltigstem Instrument der Geschichte.

Was aber ist die geschichtliche Bestimmung Europas? Sie heißt Selbstbestimmung. Selbstbestimmung war die natürliche Reaktion auf eine Lage, in der sich Europa nach seiner Teilung als Objekt Größerer wieder fand. Selbstbestimmung wurde als Ziel schon in einer Zeit formuliert, als sie sich auf den eigenen Weg zur wirtschaftlichen Bündelung seiner Kräfte beschränkte, weil sicherheitspolitisch die Abhängigkeit von der amerikanischen Macht vollständig war. Westeuropa lebte als Protektorat unter dem Schutz der Amerikaner. Beide verband das gemeinsame Interesse an Stabilität. Der Status quo im alten Kontinent sollte auch aus der Sicht der Sowjetunion gewahrt und zementiert werden. Es gab nur einen Ort, nämlich Deutschland, das damit auf Dauer nicht zufrieden sein konnte. Der schwächste Faktor entwickelte die Idee vom Gewaltverzicht. Gewaltverzicht 
erschien allen vier Siegermächten als risikolos, weil damit alle bestehenden Grenzen im geteilten Europa bestätigt wurden, und nur noch in beiderseitigem Einverständnis geändert werden durften. In keiner der vier Hauptstädte konnte man sich den gewaltigen Prozess vorstellen, der die Landkarte Europas verändert hat.

Sie wurden auf diese Weise zu Komplizen für die Schaffung einer neuen Stabilität in Europa. Die gegenseitige Bedrohung mit der größten Dichte von Streitkräften auf dem Globus konnte abgeschafft werden. Aus Gegnern wurden Partner der gemeinsamen Sicherheit. Reagan, Bush und Gorbatschow beseitigten also die atomaren Raketen mittlerer und kurzer Reichweite, die chemischen Waffen und einigten sich auf die kontrollierbare gewaltigste konventionelle Reduktion der Geschichte. Sie hatten die Macht, die Rechtsgrundlagen zu schaffen, auf denen Europa in Frieden leben konnte.

Seit nunmehr über 18 Jahren erfreut sich Europa dieser Stabilität. Das Fundament der gemeinsamen Sicherheit für Europa war stark genug, um die deutsche Einheit zu gestatten, um die Implosion der Sowjetunion und das Ende des Warschauer Paktes zu überstehen, sogar um die Ausweitung der EU, und die Ausweitung der NATO zu überdauern. Außerdem die Bildung der baltischen Staaten und die Selbständigkeit von Tschechen und Slowaken. Dieses austarierte Gebäude der Stabilität hat sogar seine Unvollständigkeit auf dem Sektor der Atomwaffen ertragen oder überlebt. Amerikanische, russische, französische und britische Atomwaffen sind Relikte aus den Zeiten der gegenseitigen Abschreckung. Zumal keine neuen atomaren Systeme auf dem Boden der nichtatomaren Staaten eingeführt wurden.

Dieses 18 Jahre lang bewährte System der Stabilität in Europa würde erschüttert, wenn Amerika seine Absicht durchsetzte, auf exterritorialen Stützpunkten in Polen und Tschechien atomare Systeme unkontrollierbarer Qualität und Zahl zu stationieren. Sie könnten Russland erreichen, mindestens zwölf Jahre bevor der Iran Raketen bauen kann, die bis Polen reichen, geschweige denn bis Amerika. Natürlich haben Washington, Warschau und Prag das Recht zu entsprechenden bilateralen Verträgen, aber sie können nicht verhindern, dass sie damit die Grundlage der Stabilität für alle Länder und Völker in Europa missachten, verletzen, gefährden, sogar zerstören. Die Bemühungen zur Lösung dieser Krise beweisen das. Wir haben dafür Zeit gewonnen, weil eine Entscheidung wohl vertagt ist, bis eine neue Administration sich im Frühsommer des nächsten Jahres eingearbeitet haben wird.

Die Grundfrage lautet: Gibt es Sicherheit mit oder gegen Russland? Durch Kooperation oder durch Konfrontation? Europäische Selbstbestimmung über die Wirtschaft hinaus ist erst eine Möglichkeit der letzten 18 Jahre geworden. Wer Konfrontation mit Russland hinnimmt oder toleriert, verhindert europäische Selbstbestimmung. Selbstbestimmung ist durch vielfachen Beschluss beschworen und beschlossen worden, aber wichtiger: Sie ist die geschichtliche Bestimmung Europas, als Ziel weder verhandelbar noch aufgebbar. Wer sie kaputt macht, verdammt Europa politisch und militärisch zum Protektorat.

Die Weichen, die in den kommenden zwölf Monaten gestellt werden, haben noch viel weiterreichende Dimensionen. Falls die Banner von Demokratie und Menschenrechten gehisst werden, egal ob in der Form eines „Bundes der Demokratien“ oder einer erweiterten NATO-Partnerschaft, wird sich zum Thema
Demokratie keine Identität zwischen Amerika und Europa entwickeln. Demokratie als Exportartikel, wenn erforderlich auch durch Gewalt verbreitet, wird keine Unterstützung bei den Völkern Europas finden und ihre Regierungen werden den Mehrheitswillen ihrer Bürgerinnen und Bürger beachten oder befolgen. Das ist Demokratie. Gleiches Recht kann dann natürlich auch nicht den Ländern verweigert werden, die Demokratie anders buchstabieren oder Demokratie nach westlichen Modellen nicht wollen. Das gilt für Russland und China, aber auch für islamisch geprägte Staaten, wie Saudi-Arabien und den Iran. Sie pochen auf ihre Rechte und haben sogar Macht.

Anders verhält es sich beim Thema der Menschenrechte. Hier findet sich eine Nähe der atlantischen Wertvorstellungen, die geschichtlich gewachsen ist und in der Charta der Vereinten Nationen 1945 formuliert wurde. Die Freiheit von Not und die Freiheit von Furcht drückt in dieser Reihenfolge eine Rangfolge aus: Nur wer nicht verhungert, kann für mehr Rechte kämpfen. Der Erfolg der Empfehlung an die Staaten, diese Prinzipien zu beachten und zu unterstützen, haben in den mehr als 60 Jahren imponierende Erfolge und deprimierende Misserfolge gezeitigt. Jedenfalls ist es nicht gelungen, zu definieren, unter welchen Voraussetzungen die Menschenrechte den Rechten der Staaten gleichgesetzt oder sogar übergeordnet werden und das Ergebnis in der Charta zu verankern. Das Prinzip der Nichteinmischung in die inneren Angelegenheiten hat Menschenrechtsverletzungen, Verbrechen gegen die Menschlichkeit, ethnische Säuberungen nolens volens gestattet; sobald daraus Massenflucht oder Vertreibungen über Grenzen hinweg wurden, die die Sicherheit anderer Staaten berühren, war die zentrale Kompetenz der UN aufgerufen.

Der Komplex ist eine globale Herausforderung geworden. Die Diskussion seit mehr als einem Jahrzehnt mündet in den Begriff der menschlichen Sicherheit. Er stellt das Individuum und seine Rechte in den Mittelpunkt und will die Staaten verpflichten, entsprechende Vor- und Fürsorgemaßnahmen umfassend zu ergreifen. Im Falle der Verletzung wäre eine internationale Einmischung legitimiert. Unbestritten dürfte ein derartiger Eingriff gegen die nationale Souveränität nur unter Bedingungen und mit Genehmigung im Auftrage des Sicherheitsrates der Vereinten Nationen erfolgen, damit er nicht zur Erweiterung imperialer Interessen missbraucht werden kann. Selbst unter solchen Einschränkungen sagt die Erfahrung, dass die Aktion einer Gruppe von Staaten praktisch immer unter den Einfluss der führenden Macht mit ihren Interessen gerät. Außerdem ist nicht zu übersehen, wie viele Jahrzehnte es dauern würde, ehe Einigkeit darüber erreicht werden kann, dass die Vereinten Nationen den einzelnen Menschen, statt den einzelnen Staat in ihren Mittelpunkt stellen.

Dennoch: Wir mussten erleben, wie schnell Amerika die Regeln der Vereinten Nationen bricht, sich selbst legitimiert und darauf beruft, dass sogar Europa im Namen der Moral den Bruch verbrieften Rechts als „Feinheit“ betrachtet. So empörend Menschenrechtsverletzungen sind, so beschämend es ist, wie hilflos und ungerecht die Völkerfamilie darauf reagiert, so sehr wird darauf zu achten sein, dass die lautere Diskussion zur Legitimierung von Interventionen zu Gunsten des Menschenrechts nicht missbraucht wird. Europa darf nicht ungewollt zum Helfershelfer einer amerikanischen Politik werden, die neben der Verbreitung von Demokratie auch die Verbreitung der Menschenrechte zu einem Instrument ihres Interesses degradiert. 
Europa ist in einer Grundfrage seiner Identität herausgefordert. Wenn es die Werte seiner geschichtlichen Bestimmung nicht genauso als unverhandelbar behauptet wie Amerika seiner geschichtlichen Bestimmung folgt, wird es moralisch und politisch die Substanz verlieren, zu einem Pol in der multipolaren Welt werden zu wollen. Die Dimension dieses Anspruchs liegt gerade in der Unverwechselbarkeit, die einen europäischen Pol von allen anderen unterscheidet. Europa vertritt den nachweisbaren Erfolg seiner Gewaltverzichtspolitik; es hat keine territorialen Ansprüche und kann niemanden bedrohen. Es garantiert das Prinzip der Einheit in Vielfalt also das Modell der Zusammenarbeit, das Schlüsselwort für viele Probleme unseres Jahrhunderts. Und vor allem verbindet Europa hohen Lebensstandard mit einem beachtlichen Netz sozialer Sicherheit für seine Gesellschaft. Neben Amerika, Russland, China und Indien könnte dieser fünfte Pol für den Globus interessant sein, selbst wenn Brasilien und Japan ihre Geltung verlangen.

Europa könnte nicht zuletzt die verheerende Rüstungsspirale unterbrechen, die Amerika in Gang gesetzt hat. Das Thema der Rüstungsbegrenzung muss dringend wieder belebt werden. Dem System der Nichtverbreitung von Atomwaffen droht der Ruin. Die atomaren Fliegerbomben müssen aus Europa verschwinden. Die Arsenale taktischer Nuklearwaffen müssen gebändigt, die Gefahren neuer Mini-Nuks gebannt werden, das Relikt des angedrohten Ersteinsatzes aus Zeiten des Kalten Krieges hat seit Russlands Partnerschaft in der NATO keinen Platz mehr. Initiativen auf diesem Sektor würden die Lasten und Gefahren ungebremster Rüstung für die Welt verringern und sogar Geld für die dringenden Probleme von Hunger und Klimaschutz frei machen.

Über die Potenziale einer Mitgliedschaft in der multipolaren Welt verfügt Europa. Ob es den erforderlichen politischen Willen aufbringt, kann niemand sagen, solange der Vertrag von Lissabon nicht ratifiziert ist und seine Mechanismen sich nicht eingespielt haben. Sogar unabhängig davon bleibt der Aufbau des Europas der Verteidigung unsere Priorität, hat der französische Präsídent gerade erklärt. Ohne Streitkräfte, über die es selbständig verfügt, ist Selbstbestimmung auf dem Sektor der Sicherheit nicht möglich.

Nachdem das Projekt einer europäischen Verteidigungsgemeinschaft (EVG) 1954 in Paris gescheitert ist, mutet es erleichternd fast wie eine historische Korrektur an, wenn nun in Paris die Führung dieser unerlässlichen Integration und in der EU angekündigt wurde.

Ein organisiertes Europa der Verteidigung wäre geeignet, mit einer Singularität umzugehen, die bisher fast vollständig beschwiegen wird. Deutschland ist das einzige Land, das in seiner Verfassung die Teilnahme an einem Angriffskrieg verbietet. Der Artikel 26 wurde bei der Aufstellung der Bundeswehr beschlossen in der Gewissheit, dass die NATO nur zur Verteidigung aktiv wird und niemand sich vorstellen konnte, dass die USA einmal das Recht für sich beanspruchen würden, Krieg ohne Mandat der Vereinten Nationen zu beginnen. Der Artikel wurde ohne Diskussion in die Verfassung des vereinten Deutschlands übernommen. Die Verfassung ist für jede deutsche Regierung bindend. Sie steht über der NATO und der UN-Charta. Deutsche Streitkräfte in einem schnellen Eingreifverband der NATO stehen unter diesem Vorbehalt. Deutsche Streitkräfte in einem Europa der Verteidigung wären ein Faktor der Berechenbarkeit, dass diese europäischen Verbände nicht ohne Mandat der UN eingesetzt werden.
Die Organisation der europäischen Verteidigung ist ein Ausdruck der europäischen Selbstbestimmung auf dem Gebiet der Sicherheit. Das wäre die praktische Garantie, dass ein Europa als fünfter Pol auf Stabilität entsprechend dem internationalen Recht ausgerichtet ist. Und in der Konsequenz auch „nein“ sagen könnte im Falle einer Wiederholung des amerikanischen Angriffskrieges gegen den Irak. Die deutschen Verpflichtungen in der Allianz und in den Vereinten Nationen blieben davon unberührt.

Die Vorstellung der europäischen Verteidigung wirft die Frage ihres Verhältnisses zu Russland auf. Gorbatschows Ideen eines Europäischen Hauses der gemeinsamen Sicherheit sahen eine Organisation vor, in der die USA als unentbehrlicher Faktor in Europa und die Sowjetunion, also NATO und Warschauer Pakt zusammenwirken. Das Modell einer Europäischen Sicherheitsgemeinschaft (ESG), das noch immer interessante Ideen für Struktur und Organisation enthält, ist ähnlich konstruiert. Der russische Präsident Medwedew hat nun einen Prozess vorgeschlagen, der zu einem Gebäude der Sicherheit für Gesamteuropa führen soll. Eine neue Organisation ist in der Tat erforderlich, weil die bestehenden Bündnisse und Verpflichtungen wie UN und NATO nicht beeinträchtigt werden dürfen. Sie müssten gleiche Sicherheit für große, wie kleine Länder bringen und glaubwürdig kollektive Mechanismen, die über jeden Nichtangriffspakt hinausgehen. Was machen wir mit dem russischen Vorschlag? Zuerst sollte darüber nachgedacht werden, um dann nicht auf dem offenen Markt nachfragen zu können, um genaueres über die russischen Vorstellungen zu erfahren. Dieser Prozess wäre geeignet, eine Reihe der aktuellen Probleme wie Raketenabwehr und NATO-Erweiterung mit Abstand zu betrachten, also die Pause auszunutzen, die durch die Ablösung der jetzigen Administration in Washington ohnehin eintritt. Das Angebot zu einem ehrlichen ruhigen Gespräch über jedes Thema, auf der Grundlage gemeinsamer europäischer Werte, sollte nicht ungenutzt bleiben. Es könnte Perspektiven für die Stabilität, Sicherheit und den Frieden für unseren gemeinsamen Kontinent unter Beteiligung Amerikas eröffnen.

Amerika und Russland stellen neue Ideen der Sicherheit vor. In beiden Fällen werden neue Organisationen für notwendig gehalten. Beide Konzepte widersprechen sich fundamental: Veränderung contra Stabilität. Das Prinzip Amerikas drängt unter seiner Führung auf Transformation, auch gegen Russland; Russland bietet seine formale Bindung im gesamt-europäischen Raum an.

Die Betrachtung unter der Überschrift: „Macht, Recht, Frieden“ hat versucht, die Vergangenheit und die Gegenwart mit Folgerungen für die Zukunft aus europäischer und deutscher Sicht zu analysieren.

Das Ergebnis führt zu einer Umkehrung. An erster Stelle muss der Frieden für das militärisch schwache Europa überragend und oberstes Ziel sein und bleiben. Der Weg dahin führt über die Stärke des Rechts, die unseren Kontinent vor dem Missbrauch militärischer Stärke bewahren kann. Das leugnet nicht die Realität der einzigen Supermacht, deren militärische Uneinholbarkeit weiter wachsen wird. Glücklicherweise handelt es sich dabei um einen Verbündeten; also kann Europa sein Prinzip der Stabilität durch Recht gegenüber den hegemonialen Drang Amerikas behaupten, wenn es will. 